\title{
Constipation in infant with premature birth story may point out colonic stricture
}

\author{
Ezer S Serin \\ Department of Pediatric Surgery, Baskent University Medical Faculty, Adana, Turkey
}

\section{Dear Editor,}

Intestinal stricture is one of the late complications of necrotizing enterocolitis (NEC) in premature babies. The presentation of intestinal stricture can manifest with symptoms such as bilious vomiting, abdominal distention or constipation due to level of intestinal obstruction. Here, I aimed to emphasize a case with constipation due to colonic stricture with premature birth story. A-3-month old female was admitted with constipation and abdominal distention for 6 days. She had 30 gestational week premature birth story with $1930 \mathrm{gr}$ in weight. According to her mother she had been followed 43 days in NICU (neonatal intensive care unit) and oral feeding had been ceased due to blood in her stool about 10 days. In physical examination she was $3000 \mathrm{gr}$ in weight and the abdomen was distended but soft. The erect abdominal radiogram showed gas distention and gasless pelvis (Figure 1A). The patient had showed symptoms of intestinal obstruction of distal level such as constipation, abdominal distention and restless. The patient was estimated having clinic chart of NEC in NICU following birth. Contrast enema revealed 2 stricture sites in colon; middle of transvers colon (10 cm long) and between descending and sigmoid colon (15 cm long) (Figure 1B). She was operated, and two stricture regions of colon were resected, and 2 colo-colonic anastomoses were done (Figure 1C). Post- operative course was uneventful. She was discharged 7 days later. She was well with 11250 gr weigh in the first year of surgery. Necrotizing enterocolitis (NEC) is a severe gastrointestinal intestinal inflammatory process often effecting premature babies. It progresses with significant mortality rates in acute stage. Clinical presentations of NEC are feeding intolerance, abdominal distention, bloody stools and severe hemodynamic instability. Treatment consists of nasogastric suction, intravenous fluids, parenteral antibiotics and, in some cases surgery if intestinal obstruction or perforations are occurred $[1,2]$. But beyond acute stage, NEC has unignorable morbid complications such as short bowel syndrome and intestinal stricture which results of recovery of affected sites of ischemic intestinal segments $[1,3]$. It should be taken into consideration that in infants, who have obstructive symptoms with a history of premature birth,
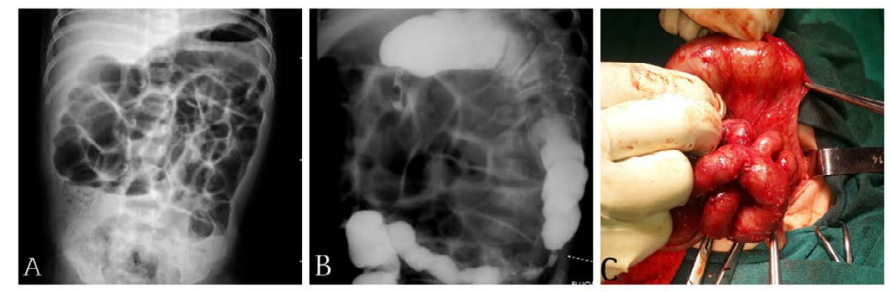

Figure 1. A. Erect abdominal radiogram showing intestinal obstruction B. Contrast enema showing strictures in transverse colon, and descending colon C. Colonic stricture during operation had developed post-NEC stricture [4]. It is reported that colon is the commonest site for stricture after NEC [5].When patients have intestinal obstruction with premature birth story, contrast enema is recommended to evaluate of colonic patency and to rule out diagnosis of stricture before operations $[1,4,5]$. It is known that if the stricture involved colonic segments patients can be asymptomatic for a while and tolerate feeding well until obstructive symptoms is occurred day by day. If prolonged constipation is not investigated patients can gradually face to high morbid complication such as intestinal obstruction, septicemia or perforation because of post-NEC strictures $[1,5]$. Colonic stricture following NEC should be index of suspicious for all clinicians who see the patients with premature birth story and with intestinal obstruction. Because the large intestine is storage organ the obstructive symptoms due to colonic stricture may not occur as early as obstructive symptoms of small intestinal stricture. I want to emphasize that colonic strictures should be stated in mind when premature birth infants present with constipation. For these reasons I wanted to explain how necessity of regular follow-up and information of family for such complications of NEC mentioned here, is important.

\section{References}

1. Karl G. Sylvester Gigi, Y. Liu, T. et al. Necrotizing Enterocolitis. In Arnold G. Coran (eds), Pediatric Surgery (seventh edition), New York. 2012. pp 187-1207.

2. Hartman GE, Drugas GT, Shochat SJ (1988) Post-necrotizing enterocolitis stricture presenting with sepsis or perforation: risk of clinical observation. J Pediatr Surg 23: 562-566. [Crossref]

3. Heida FH, Loos MH, Stolwijk L, Te Kiefte BJ, van den Ende SJ, et al. (2016) Risk factors associated with postnecrotizing enterocolitis strictures in infants. $J$ Pediatr Surg 51: 1126-1130. [Crossref]

4. Phad N, Trivedi A, Todd D, Lakkundi A (2014) Intestinal strictures post-necrotising enterocolitis: clinical profile and risk factors. J Neonatal Surg 3: 44. [Crossref]

5. Burnand KM, Zaparackaite I, Lahiri RP, Parsons G, Farrugia MK, et al. (2016) The value of contrast studies in the evaluation of bowel strictures after necrotising enterocolitis. Pediatr Surg Int 32: 465-470. [Crossref]

${ }^{\star}$ Correspondence to: Ezer S Serin, Department of Pediatric Surgery, Baskent University Medical Faculty, Adana, Turkey, Tel: 90 (533) 2583929; E-mail: semireserin@yahoo.com

Key words: constipation, colonic stricture, premature birth

Received: January 04, 2019; Accepted: January 14, 2019; Published: January 18, 2019

Copyright: (C2018 Crostelli M. This is an open-access article distributed under the terms of the Creative Commons Attribution License, which permits unrestricted use, distribution, and reproduction in any medium, provided the original author and source are credited. 